\title{
DISEÑO DE UNA RED GPON APLICADA A UN SISTEMA DE COMUNICACIONES DE BUSES DE TRANSPORTE DE ALTA VELOCIDAD (BRT)
}

\section{DESIGN OF A GPON NETWORK APPLIED TO A COMMUNICATION SYSTEM OF HIGH SPEED TRANSPORTATION BUSES (BRT)}

\author{
Javier Mansilla Yanqui'
}

RECEPCIÓN: XX DE OCTUBRE DEL 2021

ACEPTACIÓN: XX DE NOVIEMBRE DEL 2021

\section{RESUMEN}

El presente artículo tiene como propósito principal, presentary proponer el diseño de una red basada en fibra óptica como medio de transmisión, y una tecnología de acceso de telecomunicaciones denominada GPON (Red Óptica Pasiva con Capacidad de Gigabit), para la conectividad entre el Centro de Monitoreo y Gestión de red y los equipos terminales de una red de buses de transporte de pasajeros de alta velocidad (BRT), equipos conformados por: i) cámaras de video vigilancia que brindan seguridad a las personas, ii) equipos de recaudo para el control y gestión del cobro de pasajes, y iii) equipos de control de semáforos, para la programación y temporización de semáforos; todos ellos con la capacidad de conectarse a la red propuesta, mediante protocolos IP o de Ethernet.

Palabras clave: Autobuses de transporte de alta velocidad (BRT), sistema de control de semáforos, sistemas de recaudo para autobuses de transporte urbano rápido.

\begin{abstract}
The main purpose of this article is to present and propose the design of a network based on fiber optics as a transmission medium, and a telecommunications access technology called GPON (Passive Optical Network with Gigabit Capacity), for connectivity between the Network Monitoring and Management Center and the terminal equipment of a high-speed passenger transport bus network (BRT), equipment made up of: i) video surveillance cameras that provide security to people, ii) collection equipment for the control and management of the collection of tickets, and iii) traffic light control equipment, for the programming and timing of traffic lights; all of them with the ability to connect to the proposed network, using IP or Ethernet protocols.
\end{abstract}

Keywords: High Speed Transport Buses (BRT), Traffic Light Control System, Collection Systems for Rapid Urban Transport Buses. 


\section{INTRODUCCIÓN}

\subsection{Metodología adoptada}

La metodología que ha sido elegida para desarrollar el presente artículo es de tipo aplicada o tecnológica, porque su producto no es un conocimiento puro, sino tecnológico. Asimismo, surge de la necesidad de mejorar el funcionamiento de un sistema, de acuerdo con el avance de la ciencia y la tecnología de hoy en día.

\subsection{Descripción de la problemática}

El escenario donde se aplica la presente solución, corresponde al Corredor Vial Metropolitano, en el que operan buses de alta velocidad para el transporte de pasajeros en la ciudad de Lima.

Para proponer el diseño de la presente solución, se realizó una inspección a cada una de las estaciones de pasajeros, en la que se pudo verificar el estado del cableado, de los elementos pasivos y del equipamiento de la red de comunicaciones de datos, las condiciones de intemperie, el ambiente donde se encuentran instalados, así como el tiempo en que vienen operando. Estas condiciones han afectado sensiblemente su operación como sistema.

Luego de analizar el estado de la red, se evaluó una solución integral que permita superar cada uno de los problemas detectados. Asimismo, se evaluó una solución tecnológica acorde con el estado del arte y se planteó una plataforma que permita una adecuada escalabilidad de la red y la posibilidad de poder brindar nuevas prestaciones de servicio a futuro.

La solución integral elegida se basa en la elección de los componentes y elementos de red de última generación, apropiados para soportar las condiciones ambientales hostiles a las que cada elemento será sometido luego de su puesta en servicio. El diseño propuesto está basado en una topología en árbol. La transmisión de la señal es bidireccional sobre una única fibra óptica, lo que permitirá una sustancial simplificación de la red y, en consecuencia, un gran ahorro de costos.

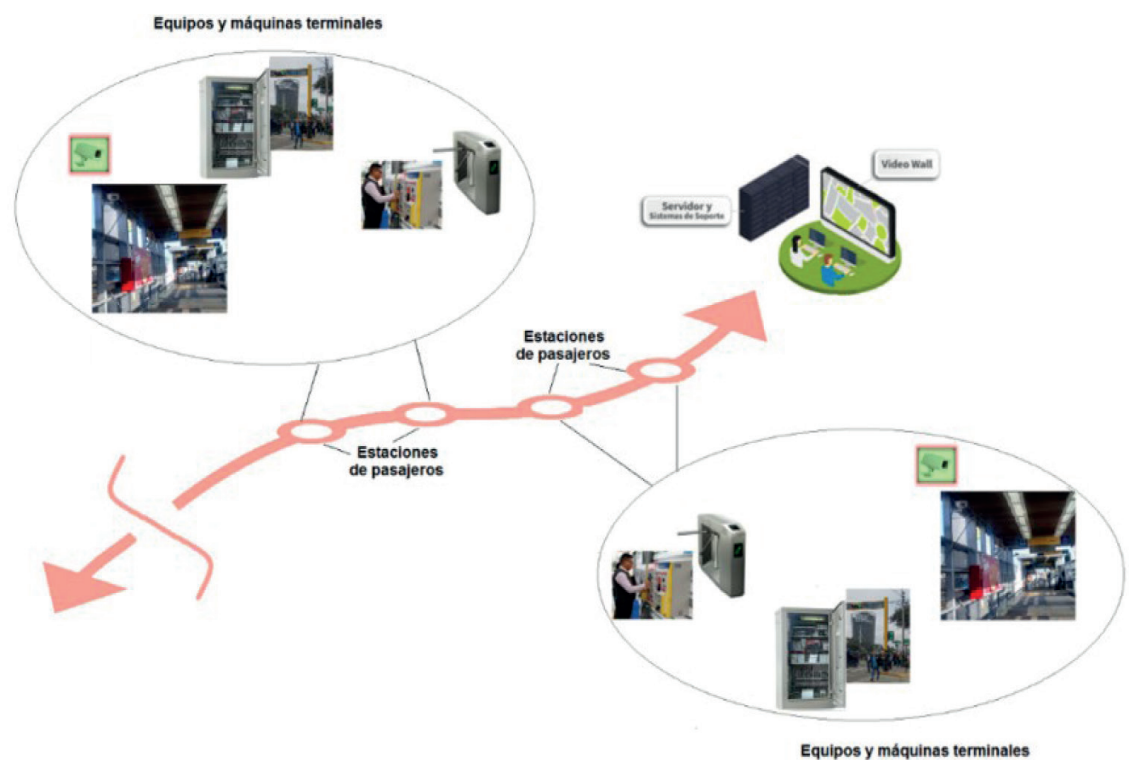

Figura 1. Concepto del sistema. Elaboración propia 


\section{CONCEPTO DEL SISTEMA}

En la figura 1, se muestra el diagrama conceptual del sistema. En la parte superior derecha, se muestra la representación simbólica del Centro de Control del Sistema. En la parte inferior derecha y superior izquierda, se observan los equipos y máquinas terminales bajo control utilizadas para brindar servicios en el Corredor Vial tales como recarga de saldo, acceso a las terrazas de abordaje, control inteligente de semáforos y video vigilancia. En la parte media central, se representan las estaciones de pasajeros del Corredor Vial.

Todos estos servicios son realizados desde el Centro de Control que cuenta con diversos sistemas de software para la Gestión y Monitoreo de dichos servicios.

\subsection{Sistemas asociados a los Servicios de Comunicaciones}

A continuación, describiremos brevemente los sistemas asociados a los servicios de comunicaciones de datos y de videovigilancia antes nombrados.

\subsubsection{Sistema de Recaudo}

Está formado por las máquinas y equipos informáticos de recaudo que permiten la recarga de tarjetas, se encuentran ubicados a la entrada de las estaciones, el acceso a las terrazas de pasajeros es a través de torniquetes de acceso donde se debita el pasaje de las tarjetas magnéticas.



Figura 2. Máquina de recarga

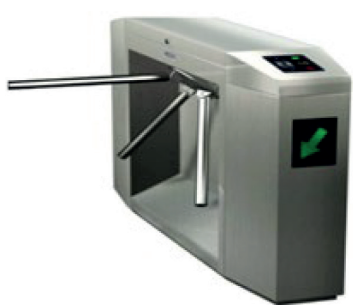

Figura 3. Torniquete de acceso

\subsubsection{Sistema de Video Vigilancia}

Está conformado por todas las cámaras que han sido dispuestas en puntos estratégicos a la entrada de la estación y en las terrazas de abordaje, para brindar protección a: i) los pasajeros, ii) los buses, y iii) bienes materiales al interior de la estación, ante eventos fortuitos como son accidentes, robos, vandalismo, peleas, etc.

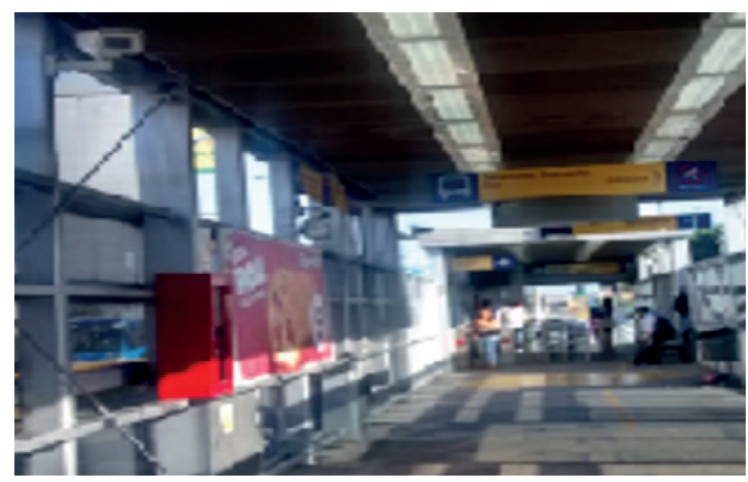

Figura 4. Cámaras de Video Vigilancia 


\subsubsection{Sistema para el control de la temporización de semáforos}

Está conformado por los equipos electromecánicos que se encargan del control de la temporización de los semáforos ubicados en los cruceros semafóricos próximos a las estaciones y en todo el trayecto de los buses.
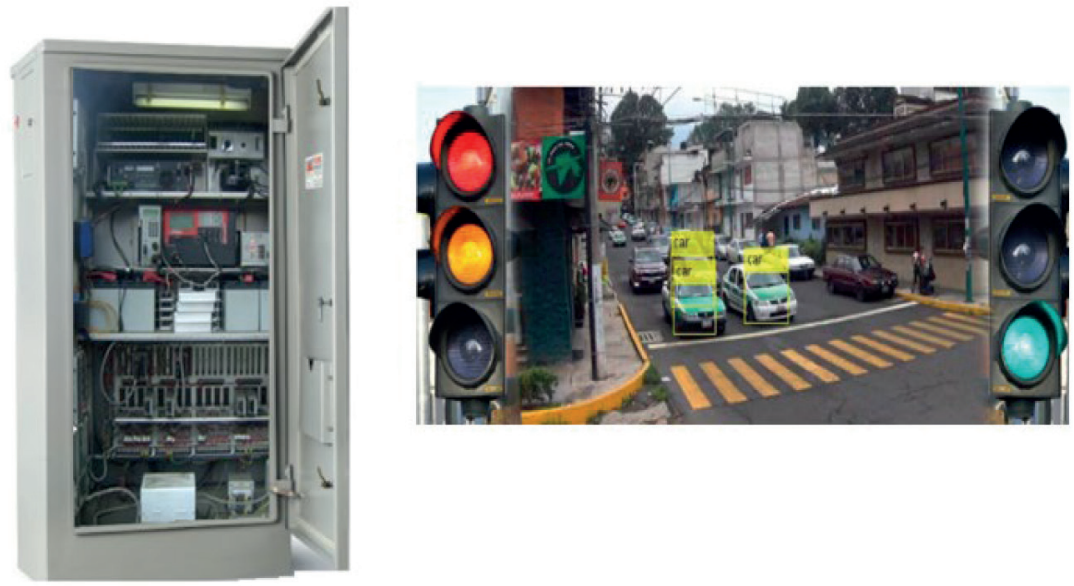

Figura 5. Controlador y semáforos de crucero

\subsubsection{Centro de control y de monitoreo}

El Centro de control se encarga de la gestión, monitoreo y mantenimiento de toda la red mediante sus sistemas de software asociados. Para el funcionamiento como sistema, se tiene implementada una red basada en equipos de conectividad enlazados a través de una red de cables de fibra óptica y soportada por una serie de elementos pasivos complementarios. Dicho centro permite que los equipos terminales brinden el servicio a los usuarios.

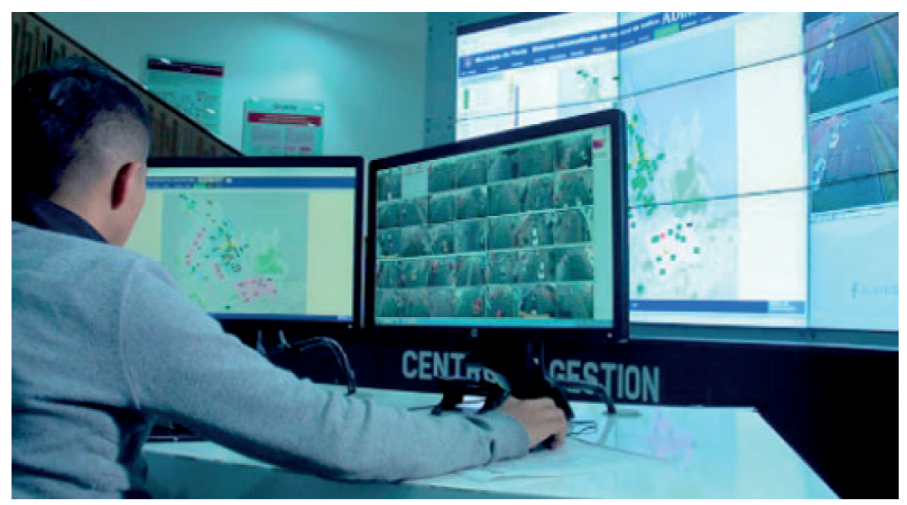

Figura 6. Centro de Control y de Monitoreo

\subsection{Red de fibra óptica basada en una topología en anillo}

La red de comunicaciones actual está basada en una topología de anillos en la que se han separado los servicios de video vigilancia y de comunicaciones de datos. 


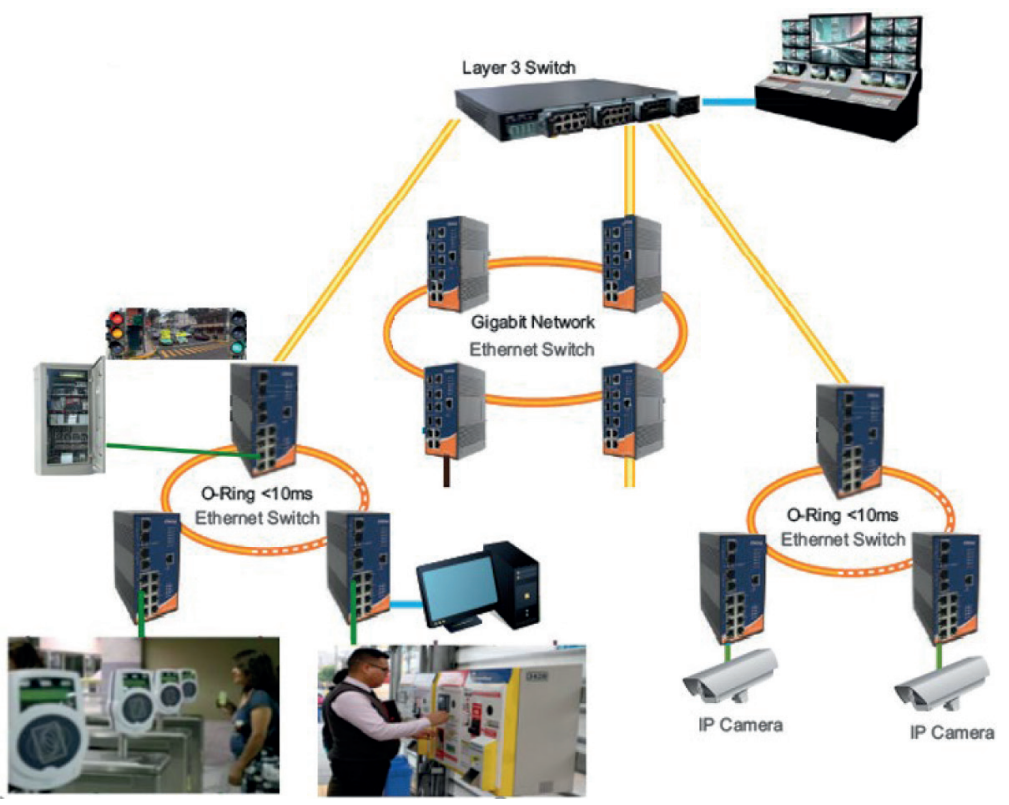

Figura 7. Diagrama simplificado de una red en anillos. Elaboración propia

En la figura 7, se observa dos grupos de anillos. En el primer grupo a la izquierda, se muestran los switches requeridos en cada estación para la prestación de los servicios de comunicaciones de datos. En el segundo grupo de la derecha, se muestra los anillos que sirven para atender los servicios de video vigilancia.

En la siguiente tabla, se muestra el resumen económico del empleo de una solución basada en una topología en anillo. En dicha solución se utilizan switches industriales producidos por un fabricante de origen israelí con presencia en Perú y a nivel mundial.

\begin{tabular}{|c|c|c|c|}
\hline \multicolumn{4}{|c|}{ ESTACIONES DEL CORREDOR VIAL METROPOLITANO } \\
\hline DESCRIPCIÓN DE LA PROPUESTA & PARCIAL & SUBTOTAL & \\
\hline I. EQUIPAMIENTO & & & S/ 3,400,290.79 \\
\hline I.1. EN LAS ESTACIONES & & S/ 2,353,027.65 & \\
\hline COMUNICACIONES & $s / 1,095,976.18$ & & \\
\hline VIDEOVIGILANCIA & $\$ / 1,257,051.47$ & & \\
\hline 1.2. EN CONTROLADORES DE SEMÁFOROS & & S/ 1,047,263.13 & \\
\hline III. INFRAESTRUCTURA & & & S/ 517,781.13 \\
\hline II.1. EN ESTACIONES & & S/ 462,897.71 & \\
\hline ELEMENTOS DE FIBRA ÓPTICA & $\$ / 39,036.18$ & & \\
\hline ELEMENTOS DE COBRE & $S / 61,156.67$ & & \\
\hline GABINETES & $S / 362,704.86$ & & \\
\hline II.2. EN CONTROLADORES DE SEMÁFOROS & & S/ 54,883.42 & \\
\hline III. OTROS & & & S/ $239,788.70$ \\
\hline TOTAL & TRUCTURA S/ & & S/ 4,157,860.62 \\
\hline
\end{tabular}

Tabla 1. Propuesta de solución con topología en anillo para el Corredor Vial Metropolitano 


\subsection{Red de fibra óptica basada en una topología en árbol}

La alternativa tecnológica basada en una topología en árbol para reemplazar la tecnología obsoleta de la red actual, basada en una topología en anillos, tiene como importante ventaja la simplicidad de la red que es a un solo hilo. Es decir, por un hilo se transmite las señales de comunicaciones desde los equipos terminales hacia el Centro de Control y desde este hacia los equipos terminales utilizando dos longitudes de onda. Esta característica permite obtener un sustancial ahorro económico en los materiales y elementos utilizados, así como los costos de mano de obra se han reducido debido a la menor cantidad de conectorizaciones y empalmes que son necesarias en comparación con la topología en anillo. Por otra parte, los equipos terminales son sencillos y fáciles de instalar y configurar. Esto ha provocado que los costos disminuyan debido a la alta difusión de esta nueva tecnología. El equipo requerido en el nodo principal denominado OLT es de característica modular y su mantenimiento es simple. La principal desventaja de esta alternativa consiste en que, ante la falla de algún divisor de señal (splitter), toda la rama de equipos terminales bajo su atención se quedará sin servicio. Sin embargo, esta condición de falla se ha minimizado en la propuesta, debido a la inclusión de una red de respaldo que permite prever una ruta alterna para minimizar el riesgo de pérdida del servicio.

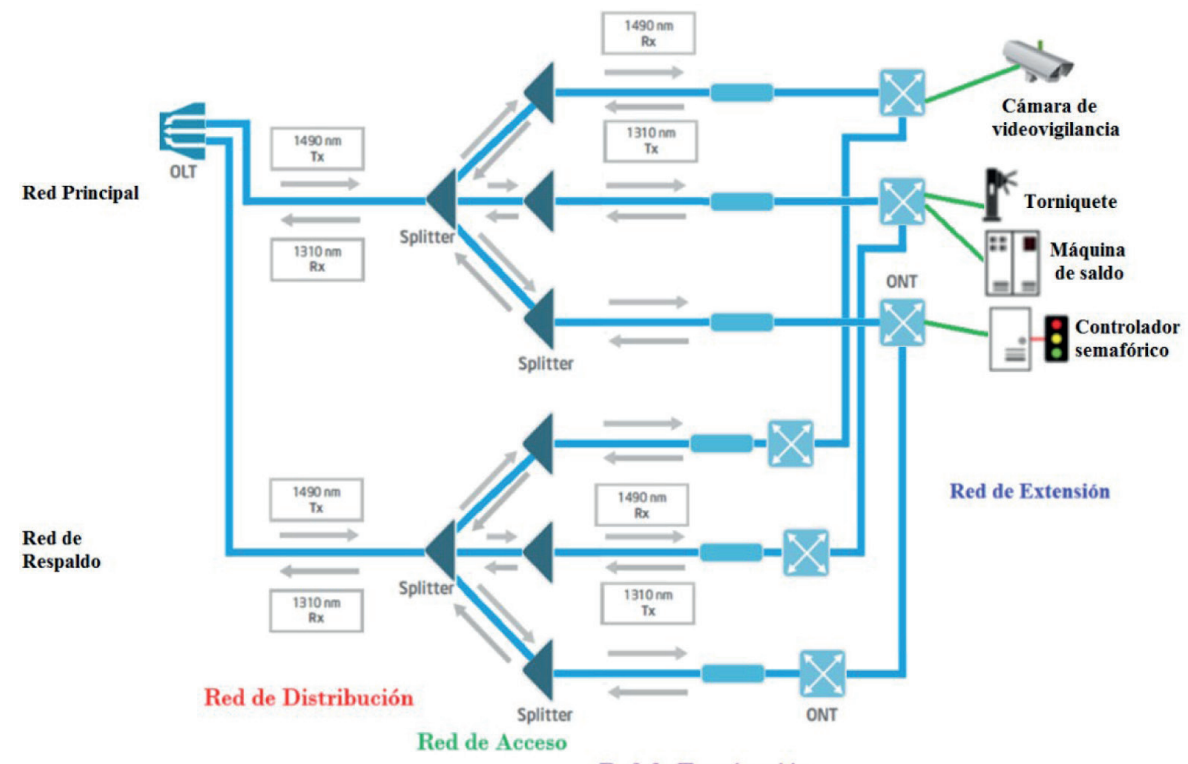

Red de Terminación

Figura 7. Diagrama simplificado de la red principal y de respaldo

\subsection{Descripción de la solución basada en una topología en árbol}

En la figura 8, se muestra la zonificación de las estaciones del Corredor Vial, para realizar el diseńo de toda la red. Desde las figuras 10 hasta la 13, se muestra el diagrama topológico en árbol dividido en cuatro zonas. Los nodos terminales que permiten la operatividad de los equipos de comunicaciones de datos y de video vigilancia están representados por círculos de diferentes colores. En la figura 9, se muestra la leyenda, los símbolos de la red como el nodo principal, los divisores (splitter) de primer y segundo nivel, los nodos terminales de la estación de pasajeros, los equipos terminales y de los medios de transmisión (cables de fibra óptica y de par trenzado en cobre). 
En el diagrama topológico, se observa la disposición de los nodos terminales y de los divisores de segundo nivel. Los colores de los nodos terminales permiten diferenciar los servicios de comunicaciones de datos de los de video vigilancia. Se ha representado y diagramado para cada una de las 4 zonas. Los enlaces que comunican los divisores de primer nivel con los de segundo nivel se aprecian con líneas de color azul y se ha especificado también sus distancias aproximadas. Por otra parte, se ha representado con líneas del mismo color a las acometidas que comunican los nodos terminales con los divisores de segundo nivel. Los divisores de segundo nivel permiten la conectividad de los nodos terminales con el nodo principal a través de los divisores de primer nivel.

Los círculos de color amarillo representan a los nodos terminales que brindan servicio a las máquinas de recaudo, para la recarga de las tarjetas magnéticas de los usuarios del servicio, y el debitado del pasaje en los torniquetes para el ingreso de usuarios a las terrazas de abordaje de pasajeros. Por su parte, los círculos de color verde representan a los nodos terminales que brindan servicio a cámaras de video vigilancia y los de color rojo representan a los nodos que alimentan a las máquinas denominadas Controladores de Semáforos para el control inteligente de semáforos.

ZONA 1: Desde la estación Matellini hasta la estación Ricardo Palma ZONA 2: Desde la estación Angamos hasta la Estación Central ZONA 3: Desde la estación Colmena hasta la estación UNI

ZONA 4: Desde la estación Honorio Delgado hasta la estación Naranjal

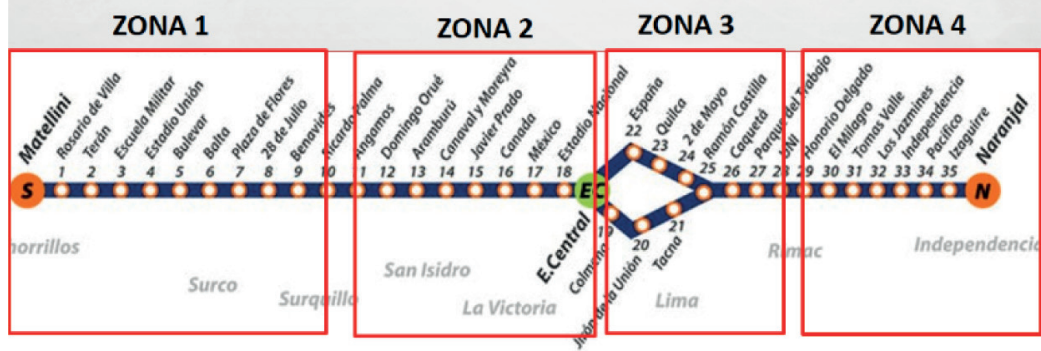

Figura 8. Zonificación de estaciones

\begin{tabular}{|c|c|c|c|}
\hline SÍMBOLOS & DESCRIPCION & SIMMBOLOS & DESCRIPCIÓN \\
\hline & Nodo Principal (OLT) & & Camara \\
\hline & $\begin{array}{c}\text { Splitter ler Nivel } \\
1: 8\end{array}$ & \begin{tabular}{|l|l|}
$\pi$ & 0 \\
& \\
\end{tabular} & $\begin{array}{c}\text { Maquina de Saldo - } \\
\text { Taquilla }\end{array}$ \\
\hline & $\begin{array}{c}\text { Splitter 2do Nivel } \\
1: 16\end{array}$ & & Torniquete \\
\hline & $\begin{array}{l}\text { Nodo terminal de } \\
\text { semaforización }\end{array}$ & & $\begin{array}{l}\text { Controlador } \\
\text { Semaforico }\end{array}$ \\
\hline & $\begin{array}{l}\text { Nodo terminal de } \\
\text { video vigilancia }\end{array}$ & & $\begin{array}{l}\text { Fibra optica } \\
\text { Monomodo }\end{array}$ \\
\hline & $\begin{array}{c}\text { Nodo Terminal } \\
\text { de recaudo }\end{array}$ & & $\begin{array}{l}\text { Red UTP } \\
\text { Cat } 6\end{array}$ \\
\hline
\end{tabular}

Figura 9. Leyenda de Diagrama Topológico 


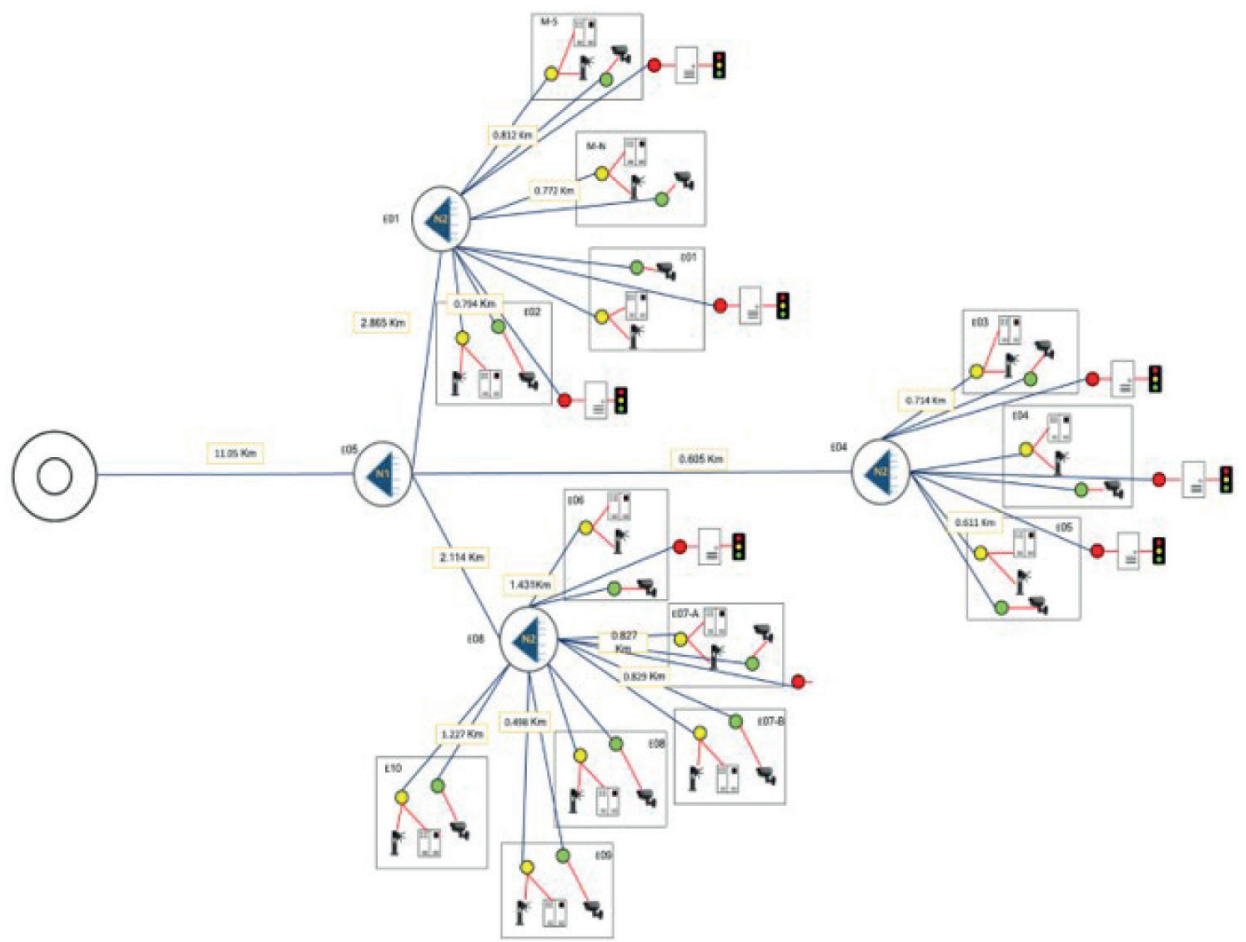

Figura 10. Diagrama Topológico de la Zona 1 (red principal)

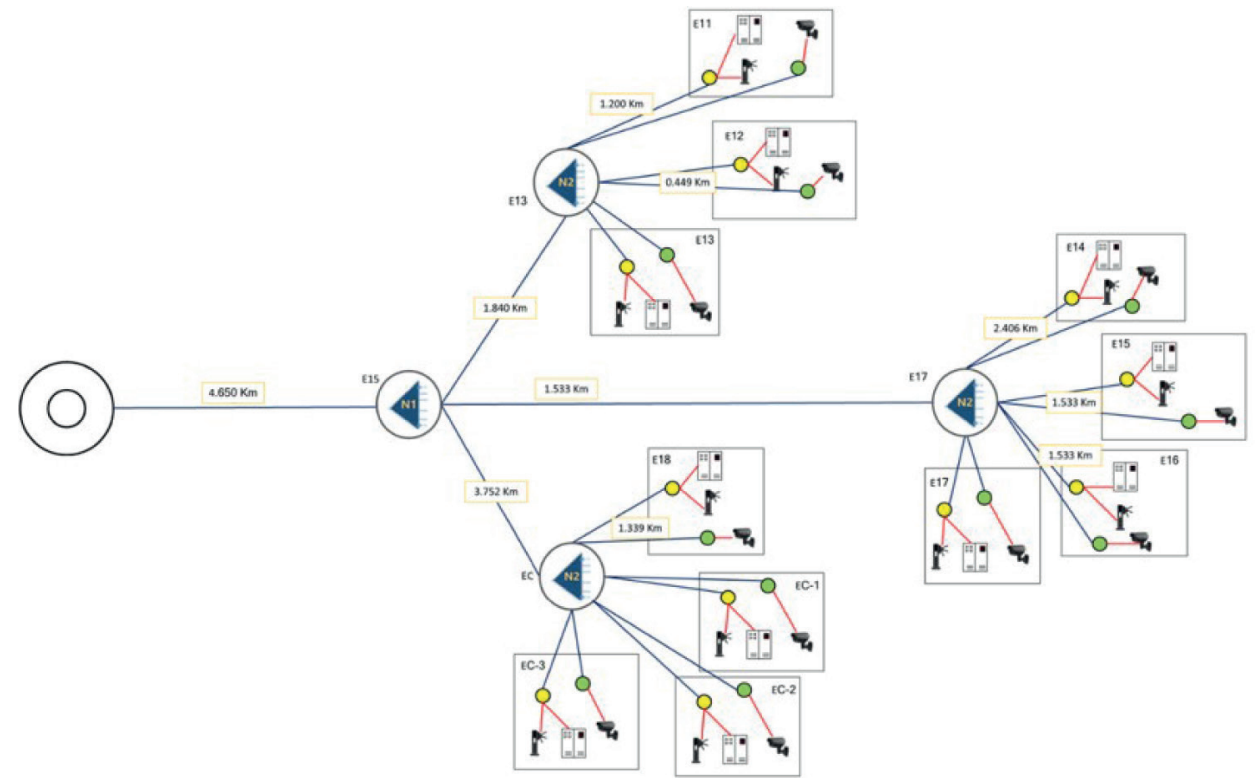

Figura 11. Diagrama Topológico de la Zona 2 (red principal) 


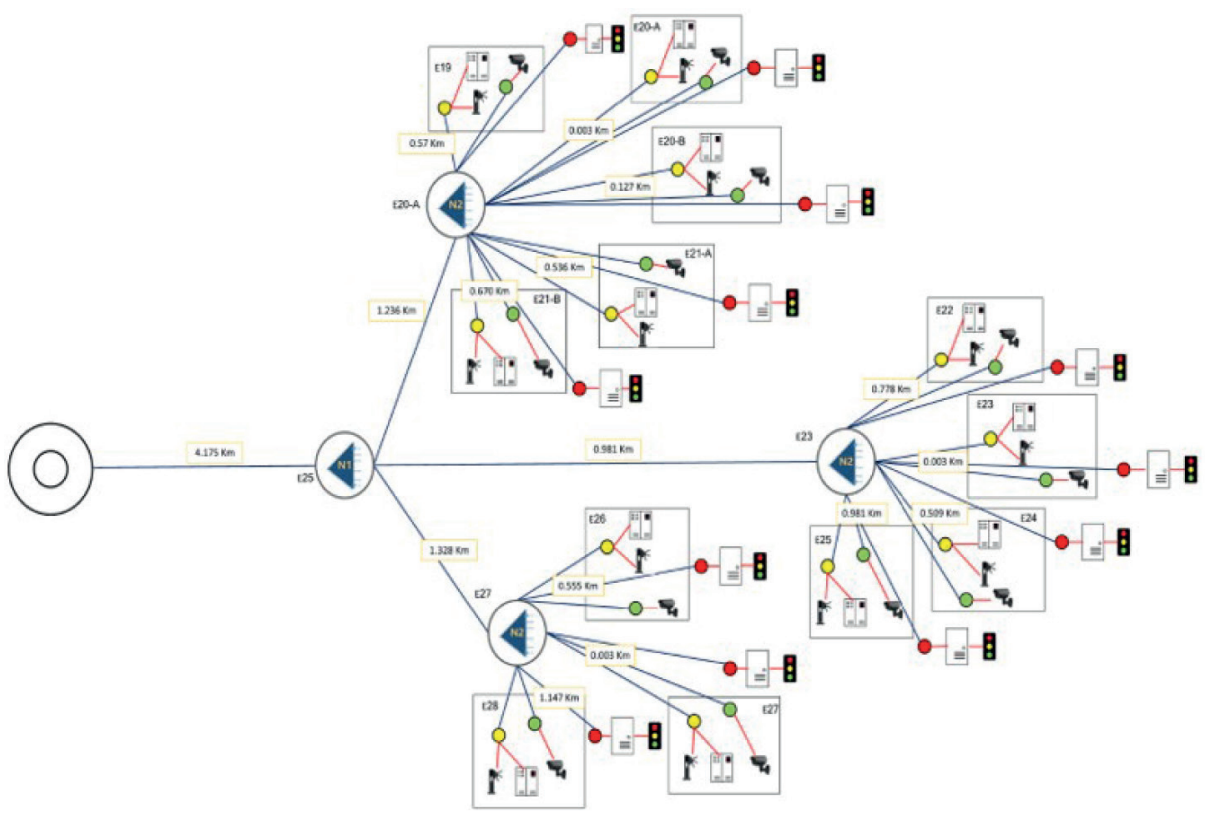

Figura 12. Diagrama Topológico de la Zona 3 (red principal)

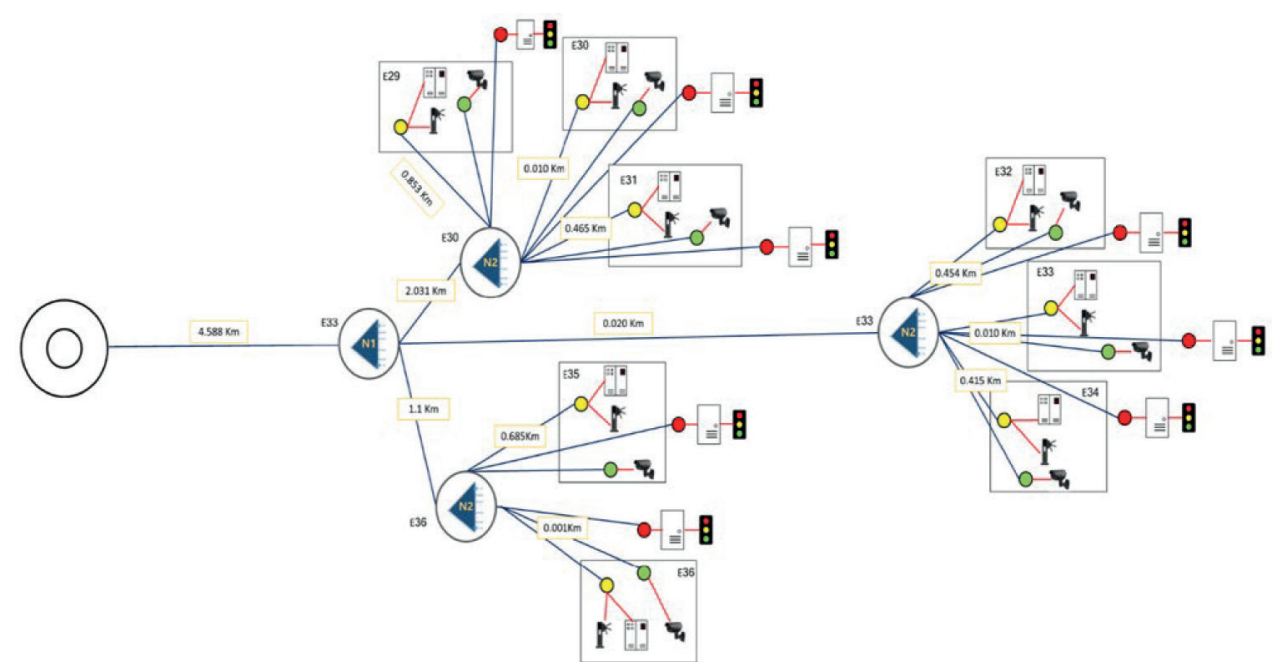

Figura 13. Diagrama Topológico de la Zona 4 (red principal)

\section{INGENIERÍA DE DETALLE DE LA SOLUCIÓN}

Para poder comprender cómo ha sido diseñada la red y su funcionamiento, se mostrará y se explicará el diseño de detalle de las redes en sus principales etapas.

En la ubicación del Centro de Control, se ha propuesto un equipo de Línea Terminal Óptico (OLT) de arquitectura modular como núcleo de la Red. La ventaja de este equipo es su crecimiento modular; 
es decir, puede crecer gradualmente conforme se incrementen las estaciones de pasajeros o se amplíe la cantidad de nodos terminales. Las tarjetas GPON se pueden cambiar en operación en caso de falla sin necesidad de apagar el equipo. Asimismo, la administración y mantenimiento preventivo de la red GPON y de los equipos terminales de red (ONT) es sencilla, gracias al software de monitoreo que posee y la simplificación de los procedimientos de instalación alcanzados con la evolución de las redes GPON.

Este software de monitoreo permite la visualización de los dispositivos de la red, su actividad, su inactividad y bloqueo, la carga del CPU y la ocupación de memoria, el tráfico cursado en la red, velocidad de transmisión máxima, promedio y mínima, datos de los dispositivos (slot, ID, estado, MAC). Esta información facilita la gestión de la red y permite reducir el tiempo de respuesta del personal de soporte ante alguna falla que se produzca. Los parámetros que se pueden visualizar son estado de ONT, potencia óptica, estado de interfaz, temperatura instantánea del transceptor, distancia, registros históricos, etc.

La solución propuesta soporta ITU-T G.984.4 para gestión y control de Interface de la ONT (OMCI).
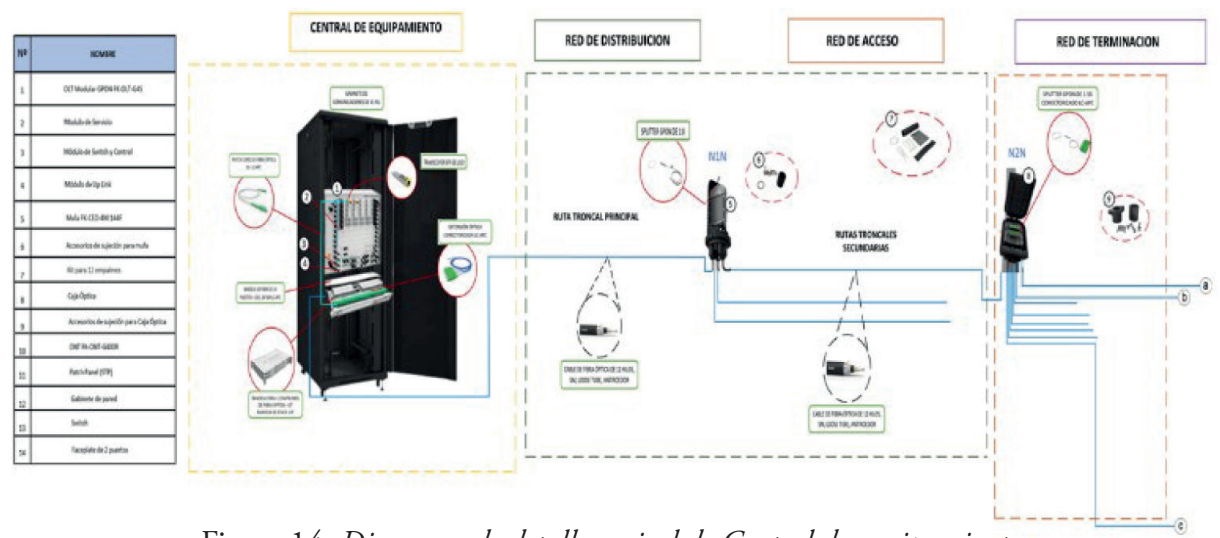

Figura 14. Diagrama de detalle a nivel de Central de equipamiento, Red de Distribución, Red de Acceso

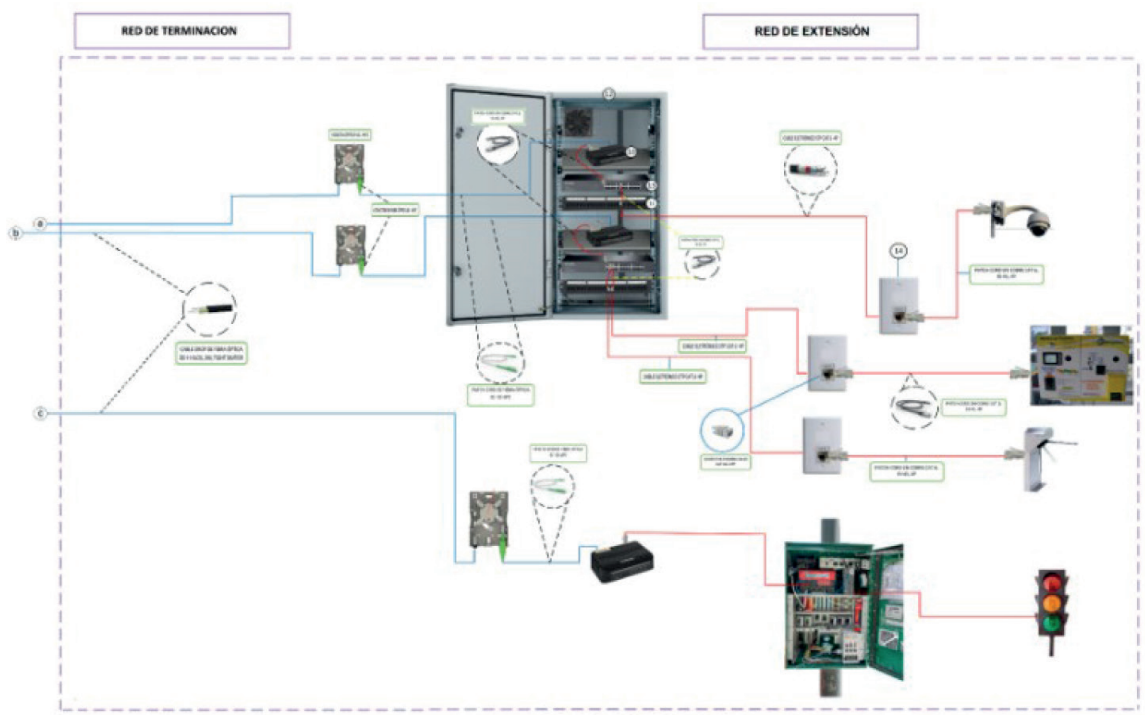

Figura 15. Diagrama de detalle a nivel de Red de Terminación y Red de Extensión 


\section{DIMENSIONAMIENTO DE LA RED PROPUESTA}

Para la implementación de la red propuesta, es necesario dimensionarla, es decir, definir las cantidades de materiales como cables, elementos pasivos y equipos que serán necesarios. En vista del tamańo de la red bajo estudio, principalmente por la cantidad de elementos por estación, fue necesario dividirla en cuatro (04) zonas para su mejor entendimiento y desarrollo.

Con este propósito, es necesario asociar el diagrama topológico de la figura 9 con la ingeniería de detalle antes mostrada. Luego de definir las cantidades de elementos, esta información se registra en la denominada matriz de dimensionamiento. El diagrama topológico es una herramienta extremadamente importante, debido a que permite una representación simplificada de toda la red bajo estudio. Gracias a ello, es posible definir las características y las cantidades de los elementos de red para determinar los datos parciales y globales.

En el diagrama topológico de la Zona 2 (figura 10), en el recuadro de la parte superior central, se muestran agrupaciones de nodos terminales correspondientes a 4 estaciones (Matellini Sur y Norte, E01 y E02). Estas agrupaciones permiten la atención de los nodos desde los divisores de segundo nivel identificados como N2. Finalmente, se muestran los enlaces (incluidas sus longitudes), que interconectan los divisores de segundo nivel con los divisores de primer nivel.

Mediante la matriz de dimensionamiento, se ha determinado las cantidades totales de los elementos pasivos, los materiales y equipos necesarios.
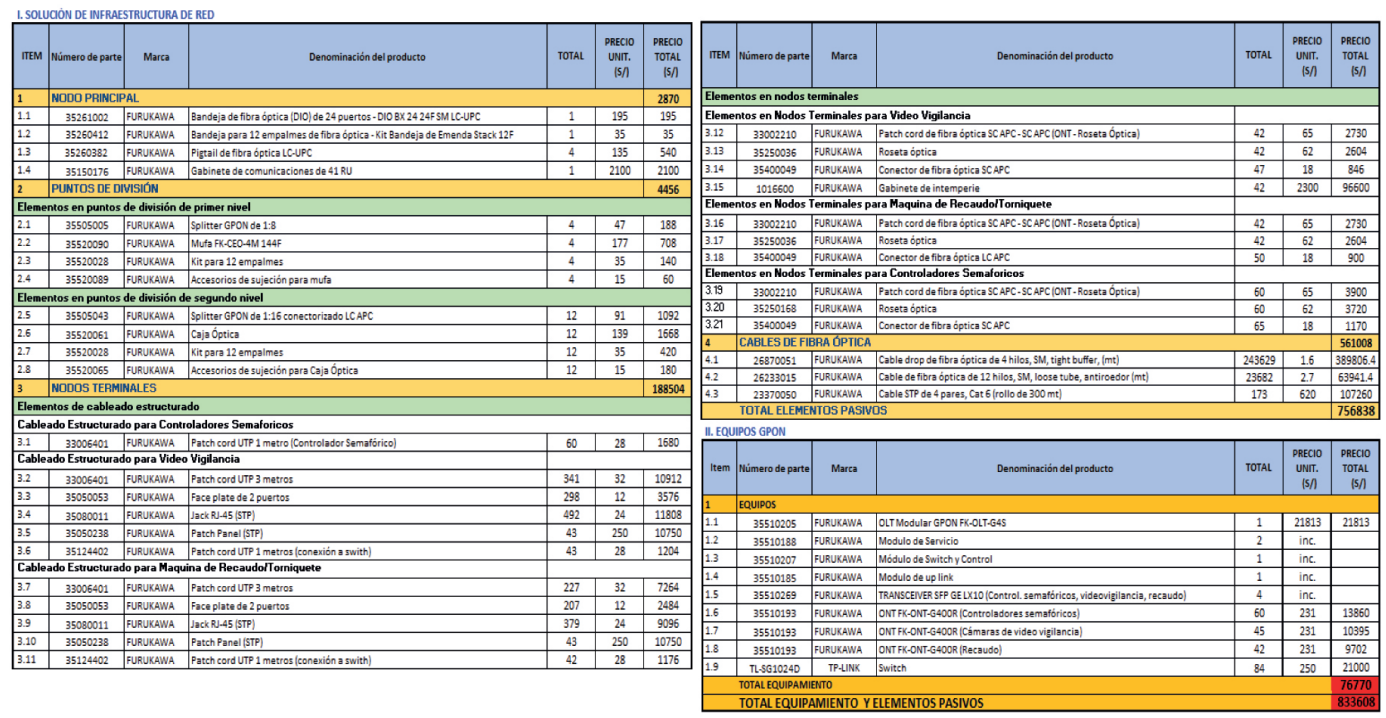

Tabla 2. Matriz de equipos, elementos pasivos y materiales

\section{EVALUACIÓN DE LAS ALTERNATIVAS TECNOLÓGICAS PROPUESTAS}

Se han evaluado dos alternativas tecnológicas para elegir la propuesta para la nueva red. La primera de ellas está basada en el concepto de la topología en anillos, que corresponde a la plataforma de comunicaciones actual, y la segunda está basada en el concepto de la topología en árbol. En el siguiente cuadro, se resumen las ventajas y desventajas de cada una de estas alternativas para finalmente concluir en la elección de una de ellas. 


\begin{tabular}{|c|c|c|c|c|}
\hline TECNOLOGÍA ACTUAL DE LA RED & COMENTARIOS & TECNOLOGÍA PROPUESTA & \multicolumn{2}{|c|}{ COMENTARIOS } \\
\hline \multirow[t]{6}{*}{ 1. Topología basada en anillos } & $\begin{array}{l}\text { a) Escalamiento. Bajo y costoso, requiere } \\
\text { de adición de equipamiento en las } \\
\text { partes intermedias de la red. }\end{array}$ & \multirow[t]{4}{*}{ 2. Topología en árbol } & a) & $\begin{array}{l}\text { Escalamiento. Fácil y de bajo costo debido a } \\
\text { que solo requiere adicionar elementos } \\
\text { activos en los extremos de la red, en las } \\
\text { partes intermedias todos los elementos son } \\
\text { pasivos. }\end{array}$ \\
\hline & $\begin{array}{l}\text { b) Instalación y ampliación de red. } \\
\text { La instalación de una nueva red es } \\
\text { compleja debido a la mayor cantidad } \\
\text { hilos operativos, de materiales por lo } \\
\text { que encarece la solución }\end{array}$ & & b) & $\begin{array}{l}\text { Instalación y ampliación de red. La } \\
\text { instalación de una nueva red o su } \\
\text { ampliación es sencilla. Por ejemplo, es } \\
\text { factible contar con cables pre- } \\
\text { conectorizados hechos en fábrica que } \\
\text { facilitan las labores de instalación y } \\
\text { abaratan los costos de mano de obra. }\end{array}$ \\
\hline & $\begin{array}{l}\text { c) Mantenimiento. Complejo. } \\
\text { Hay elementos activos en las partes } \\
\text { intermedias de la red que requieren } \\
\text { de personal especializado. }\end{array}$ & & d) & $\begin{array}{l}\text { Mantenimiento. Sencillo, todos los } \\
\text { elementos intermedios de la red son } \\
\text { pasivos }\end{array}$ \\
\hline & $\begin{array}{l}\text { e) Personal de Instalación. } \\
\text { Requiere de personal con alta } \\
\text { especialización. }\end{array}$ & & f) & $\begin{array}{l}\text { Personal de Instalación. El personal no } \\
\text { requiere de amplia experiencia. }\end{array}$ \\
\hline & $\begin{array}{l}\text { g) Tecnología utilizada. } \\
\text { Comunicación a dos hilos. Está } \\
\text { siendo abandonada por los } \\
\text { fabricantes. }\end{array}$ & & h) & $\begin{array}{l}\text { Tecnología utilizada. Se utiliza un solo hilo } \\
\text { para transmitir y recibir. Tecnología madura } \\
\text { y emergente. }\end{array}$ \\
\hline & $\begin{array}{l}\text { i) Integración de servicios. No } \\
\text { es amigable. }\end{array}$ & & j) & Integración de servicios. Fácil \\
\hline
\end{tabular}

Tabla 3. Cuadro comparativo de topologías expuestas

En conclusión, por las razones antes mostradas desde el puntos a) hasta el f) de la tabla 2, se puede afirmar que la alternativa con topología en árbol resulta la más conveniente para el diseño de la nueva red.

\section{BENEFICIOS QUE APORTA EL PROYECTO}

Los beneficios que aporta el presente proyecto se describen a continuación.

Costo Económico. En las tablas 1 y 2, se aprecia de manera aproximada los costos parciales y totales de las propuestas basadas en las topologías en anillo y en árbol. Se puede apreciar que la segunda topología es sustancialmente más económica comparada con la primera. Esto se debe a que la red con topología en árbol es totalmente pasiva; es decir, no hay equipamiento que tenga que instalarse entre el nodo principal y los nodos terminales, lo cual reduce sustancialmente los costos. Por otra parte, debido a su característica en árbol y transmisión a un solo hilo, la cantidad de cable de fibra óptica requerida es menor, ya que las portadoras ópticas que transportan la información, viajan en sentidos opuestos utilizando el mismo hilo de fibra óptica. Finalmente, los gastos de mantenimiento son menores por su simplicidad y su amplia difusión en la actualidad.

Escalabilidad. Una de las mayores ventajas de este sistema es su crecimiento escalable. La red es escalable debido a que, para realizar una ampliación de red, únicamente es necesario instalar nuevos puntos de distribución primarios y secundarios, sin afectar la operatividad del servicio. Los cambios en el lado del Centro de Gestión no afectan a la red sino en el lado del equipamiento terminal. Así, la red intermedia permanece sin afectar.

Gestión. La gestión del sistema es sencilla. Mediante su software de gestión se puede identificar a los equipos en los nodos terminales que pueden estar fallando o cuando la red requiera de una ampliación debido a la demanda. 


\section{CONCLUSIONES}

El diseño de la plataforma de comunicaciones antes descrita comprende la propuesta: a) del equipamiento y elementos pasivos a ser instalados en los gabinetes de los cuartos de comunicaciones de las estaciones de pasajeros, b) del equipamiento y elementos pasivos a ser instalados en los armarios que controlan la temporización de los semáforos, y c) del cableado de fibra óptica y cobre, el cual mejorará significativamente la red de datos de recaudo, de video vigilancia y de control semafórico del Sistema de Comunicación de Datos actual.

La nueva documentación e identificación permitirán una adecuada administración del nuevo Sistema de Comunicación de Datos.

Un nuevo Plan de Mantenimiento prevendrá las fallas del Sistemas de Comunicación de Datos.

\section{BIBLIOGRAFÍA}

[1] E. Costa, "Design of a Network Access Based on FTTH", master thesis, Universitat Politécnica de Catalunya, 2014. [Online]. Available: https://bit.ly/3qtPB1Q [Accessed: Jun 12, 2014]

[2] D. Rodríguez y E. Vergel Tovar, "Sistemas de transporte público masivo tipo BRT (Bus Rapid Transit) y desarrollo urbano en América Latina”. Land Lines, vol. 25, no. 1, pp. 16-24, ene. 2013 [En línea]. Disponible en: https://bit.ly/32QJXyQ [Accedido: 29-dic-2021].

[3] E. Correa, "Sistema de Recaudo Automático en Transporte Colectivo Urbano". APB Prodata, Sao Paulo, Brasil, 2006 [En línea]. Disponible en: https://bit.ly/3qwUQOl [Accedido: 29-dic-2021].

[4] L. Wright y W. Hook, Guia de Planificación de Sistemas BRT Autobuses de Tránsito Rápido. New York, NY, USA: Institute for Transportation \& Development Policy, 2010 [En línea]. Disponible en: https://bit.ly/3EBPciL [Accedido: 29-dic-2021].

[5] C. Gómez Figuera, "Uso de Sistemas de Transporte Masivo BRT en Ciudades con Problemas de Congestionamiento Vial”, tesis de pregrado, Universidad Católica Andrés Bello, 2010 [En línea]. Disponible en: https://bit.ly/3JuSiZR [Accedido: 29-dic-2021].

[6] U. Zamora, H. Campos, y J. Calderón, "Bus Rapid Transit (BRT) en ciudades de América Latina, los casos de Bogotá (Colombia) y Curitiba (Brasil)”, Quivera. Revista de estudios territoriales, vol. 15, no. 1, pp. 101-118, 2013 [En línea]. Disponible en: https://www.redalyc.org/ pdf/401/40128395007.pdf [Accedido: 29-dic-2021].

[7] A. Barroso García, "Diseńo de una red de fibra óptica para la implementación de servicios de una banda ancha en una zona de viviendas en casco urbano", proyecto fin de carrera, Universidad Politécnica de Madrid, 2012 [En línea]. Disponible en: https://bit.ly/3JtbdUH [Accedido: 29-dic-2021].

[8] J. Rosas y L. Chías, “Los BRT ¿nuevo paradigma de la movilidad urbana mundial?”, Revista de Investigaciones Geográficas, no. 103, pp. 1-14, 2020. doi: dx.doi.org/10.14350/rig.60045

[9] F. Rogelez, "Sistema de semaforización inteligente en la ciudad de Bogotá para mejorar los tiempos de recorrido del sistema Transmilenio", Traza, vol. 4, no. 7, pp. 10-29, 2013 [En línea]. Disponible en: https://bit.ly/3EGtuKF [Accedido: 29-dic-2021]. 